Bull. Austral. Math. Soc.

$53 \mathrm{C} 20$

VOL. 55 (1997) [513-515]

\title{
A SCHWARZ LEMMA FOR COMPLETE RIEMANNIAN MANIFOLDS
}

\section{Leung-Fu Cheung and Pui-Fai Leung}

\begin{abstract}
We prove a Schwarz Lemma for conformal mappings between two complete Riemannian manifolds when the domain manifold has Ricci curvature bounded below in terms of its distance function. This gives a partial result to a conjecture of Chua.
\end{abstract}

\section{INTRODUCTION}

In recent paper [2], Chua has given an interesting generalisation of the classical Ahlfors-Schwarz Lemma to conformal mappings from the hyperbolic space into a Riemannian manifold. His result is the following:

THEOREM. [2] Let $\left(M^{n}, g\right)$ be an $n$-dimensional Riemannian manifold and $\left(H^{n}, g_{0}\right)$ be the $n$-dimensional hyperbolic space of constant sectional curvature -1 . Let $f:\left(H^{n}, g_{0}\right) \rightarrow\left(M^{n}, g\right)$ be a conformal map. If $\operatorname{scal}\left(M^{n}\right) \leqslant-n(n-1)$ where scal $\left(M^{n}\right)$ denotes the scalar curvature of $M^{n}$, then $f$ is distance decreasing, that is, $f^{*} g \leqslant g_{0}$.

In the same paper [2], Chua proposed the following interesting generalisation.

Conjecture. [2] Let $\left(M_{0}^{n}, g_{0}\right)$ and $\left(M^{n}, g\right)$ be two complete n-dimensional Riemannian manifolds. Let $f:\left(M_{0}^{n}, g_{0}\right) \rightarrow\left(M^{n}, g\right)$ be a conformal map. If there exist positive constants $c$ and $k$ such that $f^{*}\left(\operatorname{scal}\left(M^{n}\right)\right) \leqslant k^{2} \operatorname{scal}\left(M_{0}^{n}\right) \leqslant-c^{2}<0$, then $k^{2}\left(f^{*} g\right) \leqslant g_{0}$.

Our purpose in this note is to prove the following partial result on Chua's Conjecture.

MAIN TheOREM. Let $\left(M_{0}^{n}, g_{0}\right)$ and $\left(M^{n}, g\right)$ be two complete $n$-dimensional Riemannian manifolds. Let $f:\left(M_{0}^{n}, g_{0}\right) \rightarrow\left(M^{n}, g\right)$ be a conformal map. Assume that $\operatorname{Ric}\left(M_{0}^{n}\right) \geqslant-a^{2}\left(1+y^{2} \log ^{2}(y+2)\right)$, where Ric $\left(M_{0}^{n}\right)$ denotes the Ricci curvature of $M_{0}^{n}, a$ denotes a constant and $y$ is the distance function from a fixed origin $x_{0} \in M_{0}^{n}$. If there exist positive constants $c$ and $k$ such that $f^{*}\left(\operatorname{scal}\left(M^{n}\right)\right) \leqslant k^{2} \operatorname{scal}\left(M_{0}^{n}\right) \leqslant$ $-c^{2}<0$, then $k^{2}\left(f^{*} g\right) \leqslant g_{0}$.

\section{Received 30 July 1996}

We would like to thank K.S. Chua for introducing us to his conjecture and for many helpful discussions.

Copyright Clearance Centre, Inc. Serial-fee code: 0004-9729/97 \$A2.00+0.00. 


\section{PROOF OF THE MAIN THEOREM}

Since $f$ is conformal, there exists a smooth function $\rho$ on $M_{0}^{n}$ such that

$$
f^{*} g=e^{2 \rho} g_{0}
$$

(See $\left[3\right.$, p.53].) Following $\left[3\right.$, p.62] we put $u=e^{-\rho}$.

Since $u \geqslant 0$, therefore $r:=\inf u$ exists.

The assumption on Ric $\left(M_{0}^{n}\right)$ allows us to apply the Generalised Maximum Principle of Chen and Xin [1, Theorem 2.2, p.359] to the function $-u$ to obtain the following:

For each positive integer $m$, there exists a point $x_{m} \in M_{0}^{n}$ such that

and

$$
\begin{aligned}
0 \leqslant r \leqslant u\left(x_{m}\right) & <r+\frac{1}{m}, \\
\left|\nabla u\left(x_{m}\right)\right|^{2} & <\frac{1}{m}, \\
\Delta u\left(x_{m}\right) & >-\frac{1}{m} .
\end{aligned}
$$

Here $\nabla u$ and $\Delta u$ denote the gradient and the Laplacian of $u$ respectively.

Let $K_{0}=\operatorname{scal}\left(M_{0}^{n}\right)$ and $K=f^{*}\left(\operatorname{scal}\left(M^{n}\right)\right)$. Then by $[3, \mathrm{p} .62,(3.12)]$ we have

$$
K=u^{2} K_{0}+2(n-1) u \Delta u-n(n-1)|\nabla u|^{2} .
$$

Therefore

$$
k^{2} K_{0} \geqslant u^{2} K_{0}+2(n-1) u \Delta u-n(n-1)|\nabla u|^{2} .
$$

(since $K \leqslant k^{2} K_{0}$ ) and hence

$$
\begin{aligned}
\left(k^{2}-u\left(x_{m}\right)^{2}\right) K_{0}\left(x_{m}\right) & \geqslant(n-1)\left[2 u\left(x_{m}\right) \Delta u\left(x_{m}\right)-n\left|\nabla u\left(x_{m}\right)\right|^{2}\right] \\
& \geqslant(n-1)\left[2\left(r+\frac{1}{m}\right)\left(-\frac{1}{m}\right)-\frac{n}{m}\right] \\
& =-\frac{n-1}{m}\left(2 r+\frac{2}{m}+n\right) .
\end{aligned}
$$

Our Main Theorem will be proven if we can show that $r \geqslant k$. Suppose on the contrary that we have $r<k$. Then for $m$ large enough, we have

$$
u\left(x_{m}\right)<r+\frac{1}{m}<k .
$$

Since $K \leqslant k^{2} K_{0} \leqslant-c^{2}$, it follows from (2.1) that for $m$ large enough, we have

$$
\left(k^{2}-u\left(x_{m}\right)^{2}\right)\left(-c^{2}\right) \geqslant-\frac{n-1}{m}\left(2 r+\frac{2}{m}+n\right) k^{2} \text {. }
$$

Letting $m \rightarrow \infty$, we obtain

$$
\left(k^{2}-r^{2}\right)\left(-c^{2}\right) \geqslant 0
$$

and this contradicts the assumption that $r<k$. Therefore we must have $r \geqslant k$. 


\section{REFERENCES}

[1] Q. Chen and Y.L. Xin, 'A generalized maximum principle and its applications in geometry', Amer. J. Math. 114 (1992), 355-366.

[2] K.S. Chua, 'A generalisation of Ahlfors-Schwarz lemma to Riemannian geometry', Bull. Austral. Math. Soc. 51 (1995), 517-520.

[3] K. Yano and M. Obata, 'Conformal changes of Riemannian metrics', J. Differential Geom. $4(1970)$, 53-72.

Department of Mathematics

Hong Kong Polytechnic University

Hung Hom

Kowloon

Hong Kong

e-mail: malfcheu@hkpu02.polyu.edu.hk
Department of Mathematics

National University of Singapore

Kent Ridge

Singapore 0511

e-mail: matfredl@leonis.nus.sg 